TI 2013-049/III

Tinbergen Institute Discussion Paper

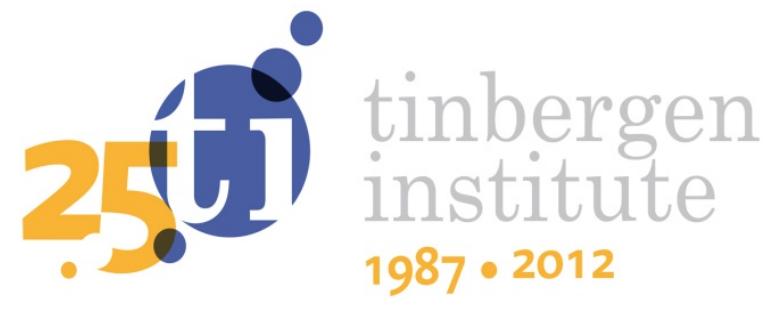

\title{
Are Forecast Updates Progressive?
}

\author{
Chia-Lin Chang
}

Philip Hans Franses ${ }^{2}$

Michael McAleer2,3,4

I National Chung Hsing University Taichung;

2 Econometric Institute, Erasmus School of Economics, Erasmus University Rotterdam, and Tinbergen Institute;

3 Complutense University of Madrid;

4 Kyoto University. 
Tinbergen Institute is the graduate school and research institute in economics of Erasmus University Rotterdam, the University of Amsterdam and VU University Amsterdam.

More TI discussion papers can be downloaded at http://www.tinbergen.nl

Tinbergen Institute has two locations:

Tinbergen Institute Amsterdam

Gustav Mahlerplein 117

1082 MS Amsterdam

The Netherlands

Tel.: +31(0)205251600

Tinbergen Institute Rotterdam

Burg. Oudlaan 50

3062 PA Rotterdam

The Netherlands

Tel.: +31(0)10 4088900

Fax: $+31(0) 104089031$

Duisenberg school of finance is a collaboration of the Dutch financial sector and universities, with the ambition to support innovative research and offer top quality academic education in core areas of finance.

DSF research papers can be downloaded at: http://www.dsf.nl/

Duisenberg school of finance

Gustav Mahlerplein 117

1082 MS Amsterdam

The Netherlands

Tel.: +31(0)20 5258579 


\title{
Are Forecast Updates Progressive?*
}

\author{
Chia-Lin Chang \\ Department of Applied Economics \\ Department of Finance \\ National Chung Hsing University \\ Taichung, Taiwan \\ Philip Hans Franses \\ Econometric Institute \\ Erasmus School of Economics \\ Erasmus University Rotterdam \\ and \\ Tinbergen Institute \\ The Netherlands \\ Michael McAleer \\ Econometric Institute \\ Erasmus School of Economics \\ Erasmus University Rotterdam \\ and \\ Tinbergen Institute \\ The Netherlands \\ and \\ Department of Quantitative Economics \\ Complutense University of Madrid \\ and \\ Institute of Economic Research \\ Kyoto University
}

Revised: March 2013

\footnotetext{
* The authors are grateful to a referee for helpful comments and suggestions. For financial support, the first author wishes to thank the National Science Council, Taiwan, and the third author wishes to thank the Australian Research Council, National Science Council, Taiwan, and the Japan Society for the Promotion of Science.
} 


\begin{abstract}
Many macroeconomic forecasts and forecast updates like those from IMF and OECD typically involve both a model component, which is replicable, as well as intuition, which is non-replicable. Intuition is expert knowledge possessed by a forecaster. If forecast updates are progressive, forecast updates should become more accurate, on average, as the actual value is approached. Otherwise, forecast updates would be neutral. The paper proposes a methodology to test whether macroeconomic forecast updates are progressive, where the interaction between model and intuition is explicitly taken into account. The data set for the empirical analysis is for Taiwan, where we have three decades of quarterly data available of forecasts and their updates of the inflation rate and real GDP growth rate. Our empirical results suggest that the forecast updates for Taiwan are progressive, and that progress can be explained predominantly by improved intuition.
\end{abstract}

Keywords: Macroeconomic forecasts, econometric models, intuition, progressive forecast updates, forecast errors.

JEL Classifications: C53, C22, E27, E37. 


\section{Introduction}

Macroeconomic forecasts like those given by the IMF and OECD typically involve both an econometric model component, which is replicable, as well as intuition, which is non-replicable. The exact balance between these two components is usually unknown to the analyst as intuition is expert knowledge only possessed by a forecaster. Macroeconomic forecasts based on an econometric model are replicable, whereas the use of intuition in providing forecasts makes the ultimate forecast non-replicable. Governments or institutions typically provide non-replicable forecasts of economic fundamentals, such as the inflation rate and real GDP growth rate, as is also the case in Taiwan, the case study in our paper. This paper concerns the analysis of sequences of forecasts for the same event, where the interest is to see if updates to the econometric model or of intuition make the joint forecasts better.

A forecast update is the difference over time between forecasts of a given variable at a finxed moment in time. The difference between the actual value and a forecast is the forecast error. Two key issues that warrant examination are: (i) whether intuition or publicly available information is more important in explaining the progress in forecast updates of economic fundamentals; and (ii) whether intuition or publicly available information are related to the forecast horizons (one-step versus multiple-step ahead forecasts).

If forecast updates are progressive, then forecasts should become more accurate, on average, as the updates approach their actual counterparts. An alternative is that forecast updates would be neutral. In this paper, the empirical application and tests of neutral forecast updates against the alternative of progressive forecast updates are based on government forecasts for Taiwan. Data for Taiwan are chosen for several reasons. First, three decades of quarterly data are available for checking the accuracy of the government forecasts of the inflation rate and the real GDP growth 
rate, and hence the effects of model versus intuition, for different forecast horizons. Second, the effects of cumulative forecast errors can be examined for accuracy, and a comparison of model and intuition. Third, the actual series for both the inflation rate and the real GDP growth rate are always released by the government one quarter immediately after the release of the revised forecast. Fourth and in stark contrast to many Western countries, the government does not revise the actual values after they have been released. Finally, macroeconomic forecasts in Taiwan are typically based on both model and intuition.

The plan of the remainder of the paper is as follows. Section 2 presents the methodology which involves simple regression models, forecasts, forecast updates, forecast errors and intuition. A key feature of our methodology is that we seek to decompose the quoted forecast into a repliacable and a non-replicable component. This decomposition is relevant for the subsequent analysis of forecast errors. Section 3 presents the data set for the empirical analysis. Section 4 analyses the empirical results. Finally, Section 5 gives some concluding comments.

\section{Methodology}

In this section we present an econometric model to obtain replicable forecasts from given forecasts and an estimate of intuition, conditional on the information set that is available to the analyst. The available forecasts can be used to compute one-period forecast updates, and one-step, two-step, three-step, and more steps-ahead forecast errors, so that the information set expands with the availability of forecasts.

Consider the variable of interest, $X_{t}$, and the availability of two sets of multi-period forecasts, $F_{1, t}$ and $F_{2, t}$, for $t=n+1, n+2, \ldots, n+N$, which the analyst aims to evaluate. Note that $F_{1, t}$ can 
be a two-step-ahead forecast and that $F_{2, t}$ can be a one-step-ahead forecast. If the forecasts are based on linear econometric models, these models may be given as

$$
\begin{aligned}
& X_{t}=W_{1, t} \beta_{1}+\varepsilon_{1, t} \\
& X_{t}=W_{2, t} \beta_{2}+\varepsilon_{2, t}
\end{aligned}
$$

When OLS is used to estimate the unknown parameters, the unbiased forecasts are given as

$$
\begin{aligned}
& F_{1, t}=W_{1, t} \hat{\beta}_{1} \\
& F_{2, t}=W_{2, t} \hat{\beta}_{2}
\end{aligned}
$$

In practice, it is likely that only the outcomes $F_{1, t}$ and $F_{2, t}$ are available, but the information sets, $W_{1, t}$ and $W_{2, t}$, are not, in which case the publicly available information set, $W_{t}$, may be used. Note that $W_{t}$ may include both $W_{1, t}$ and $W_{2, t}$, but this is unknown to the analyst. When $W_{2, t}$ nests $W_{1, t}$, such as when the one-period forecast $F_{1, t}=W_{1, t} \hat{\beta}_{1}$ is included in $W_{2, t}$, the techniques developed in Clark and McCracken [3] are useful to evaluate the quality of the forecasts. Such updating of the information set with currently available forecasts is one of the illustrative cases of forecast updates considered in this paper.

The inclusion of a two-period forecast in the information set to calculate a one-period forecast for the following period, as in (3) and (4), can be shown to contain measurement errors through the use of generated variables (see Pagan [9] and Oxley and McAleer [8]), in which case standard econometric analysis needs to be modified. The measurement error ensures that the 
covariance matrix is not proportional to the identity matrix, as the errors are serially correlated and heteroskedastic. In such cases, Franses et al. [5] demonstrate that OLS estimation can nevertheless be consistent and efficient.

Where forecasts are used to explain the variables of interest, Franses et al. [5] established the conditions under which OLS estimation of the parameters is efficient by appealing to Kruskal's Theorem, which is necessary and sufficient for OLS to be efficient (see McAleer and McKenzie [7], McAleer [6], Fiebig et al. [4], and Chang et al. [2] for further details). Moreover, as the presence of such measurement errors suggests that OLS yields biased standard errors, the Newey-West HAC standard errors should be calculated (see Smith and McAleer [10]).

\section{Data}

In this section we examine the accuracy of three sets of macroeconomic forecasts and the effects of intuition for different forecast horizons. The data are obtained from the Quarterly National Economic Trends, Directorate-General of Budget, Accounting and Statistics, Executive Yuan, Taiwan, 1980-2009. Since 1978, actual data and initial, primary and revised forecasts of the inflation rate and real GDP growth rate have been released by the Government of Taiwan, as follows (for further details, see Chang et al. [2]):

(i) In Q1 (February), release initial forecasts for Q1, Q2, Q3 and Q4 in the same year; release primary forecast for Q3 in the previous year; and release revised forecast for Q4 in the previous year;

(ii) In Q2 (May), release initial forecasts for Q2, Q3 and Q4 in the same year; release initial forecasts for Q1 and Q2 for the following year; release primary forecast for Q4 in the previous year; and release revised forecast for Q1 in the same year; 
(iii) In Q3 (August), release initial forecasts for Q3 and Q4 in the same year; release primary forecast for Q1 in the same year; and release revised forecast for Q2 in the same year;

(iv) In Q4 (November), release initial forecasts for Q4 in the same year and Q1, Q2, Q3 and Q4 in the following year; release primary forecast for Q2 in the same year; and release revised forecast for Q3 in the same year.

Thus, for one particular quarter in the future there are several forecasts for each period, namely the initial (I) forecast made in the current quarter, the primary (P) forecast that is made available one quarter later, and the revised (R) forecast that is available two quarters later. Only the initial forecast is a genuine one-quarter forecast, with both the primary and revised forecasts being revisions of the initial forecast. In sum, there are three types of forecasts of our interest. There is the initial forecast, which is the first forecast for a particular quarter. Then there is the primary forecast, which is the one quarter update of an initial forecast. The revised forecast is a one-quarter update of a primary forecast. Finally, one quarter after the revised forecast is made available, the government reports the actual values of the variables, against which the accuracy of the initial, primary and revised forecasts, as well as the effects of intuition.

In the context of equations (3) and (4), the initial forecast and the primary forecast, which includes the initial forecast in the updated information set, are such that $\mathrm{I}=F_{1, t}$ and $\mathrm{P}=F_{2, t}$. When the primary forecast is included in the updated information set to obtain the revised forecast, it follows that $\mathrm{P}=F_{1, t}$ and $\mathrm{R}=F_{2, t}$.

The variables P-I and R-P denote one-quarter forecast updates, A-R denotes a one-quarter forecast error, A-P (= A-R + R-P) denotes a two-quarter forecast error, and A-I (= A-R + R-P + P-I) denotes a three-quarter forecast error. An analysis of A-R, A-P and A-I permits a 
comparison of the effects of intuition of the expert forecaster versus the usefulness of publicly available information over forecast horizons of one, two and three quarters.

The sample period used for the actual values and the three sets of government forecasts of seasonally unadjusted quarterly inflation rate and real growth rate of GDP is 1980Q1 to 2009Q2, for a total of 118 observations. Actual data on the inflation rate and real growth rate are used in the empirical analysis to evaluate the quality of the initial, primary and revised forecasts.

\section{Empirical Results}

We have analyzed the data on unit roots and structural breaks. The diagnostics for unit roots (which are unreported) indicate that we can work with the growth rates data, as in Figures 1 and 2. Visual inspection from the same graphs does not suggest potential structural breaks, and there is also no evidence of structural breaks caused by any changes in measurement methods at the government agency in Taiwan.

The initial, primary and revised forecasts of the inflation rate, as well as the actual data, are given in Figure 1, while the real GDP growth rate counterparts are shown in Figure 2. Both figures show that the actual data, and initial, primary and revised forecasts of the inflation rate and real growth rate, are reasonably similar, with most turning points being forecast accurately. The correlations among the three forecasts and the actual values for the inflation rate and real GDP growth rate are given in Tables 1 and 2. It is clear that the four variables are highly correlated, with the lowest correlations being the initial forecasts and the actual values for both the inflation rate and real GDP growth rate, and the highest correlations being the revised forecasts and actual values of the inflation rate and real GDP growth rate. The primary and revised forecasts are very highly 
correlated, namely 0.999 for the inflation rate, and 0.994 for the real GDP growth rate, suggesting only small updates in between these forecasts.

The similarities in the three forecasts and actual values of the inflation rate and real GDP growth rate can also be seen in the root mean squared prediction errors (RMSPE) and mean absolute deviations (MAD) in Table 3. On the basis of both goodness-of-fit measures, it is clear for both the inflation rate and the real GDP growth rate that the revised forecast is more accurate than the primary forecast which, in turn, is more accurate than the initial forecast. However, the primary and revised forecasts are very similar, especially for the inflation rate, as can also be seen from the correlations in Tables 1 and 2.

The one-quarter forecast updates, namely P-I and R-P, one-quarter forecast errors, A-R, two-quarter forecast errors, A-P, and three-quarter forecast errors, A-I, are given in Figures 3 and 4 for the inflation rate and real GDP growth rate, respectively. These five variables are generally not highly correlated, as can be seen in Tables 4 and 5. The two exceptions for both the inflation rate and real GDP growth rate are between the pairs (A-R, A-P) and (A-I, P-I). The first pair is highly correlated as the primary and revised forecasts are highly correlated, while the second pair is highly correlated because A-I = (A-R) + (R-P) + (P-I), and P-I has low correlations with both A-R and R-P.

A comparison of intuition versus publicly available information in explaining forecast updates is given in Table 6 for the inflation rate and real GDP growth rate. In this table we examine the regression of forecast updates on observable explanatory variables. All that is left could be called intuition. If publicly available information is able to predict the one-quarter forecast updates, intuition would be less important than the updates of econometric models. 
The publicly available information (and available to the analyst) that is used to forecast the one-quarter forecast updates includes the one- and two-quarter lagged values of both the inflation rate and real GDP growth rate. It can be seen from Table 6 that the one-quarter forecast update, R-P, for the inflation rate and real GDP growth rate, cannot be explained using publicly available information. However, P-I (though only at the $10 \%$ level for the inflation rate) can be explained using publicly available information, specifically lagged values of the respective variables. Thus, publicly available information is significant in forecasting the one-quarter forecast update, P-I, for both the inflation rate and the real GDP growth rate, while intuition alone is paramount in explaining the one-quarter forecast update, R-P, for both variables.

Moreover, on the basis of taking the adjusted $R^{2}$ value as capturing the model contribution to goodness-of-fit and the remainder coming from intuition, Table 6 shows that updates for the inflation rate are 0.958/0.042 for intuition versus model for the P-I update, and 0.978/0.022 for the R-P update. For the real GDP growth rate, the ratios of intuition to model are $0.687 / 0.313$ and 0.978/0.022 for P-I and R-P, respectively. Combining these results with the RMSPE and MAD statistics in Table 3 suggests that forecast accuracy can be improved more substantially when forecast updates are based more heavily on a replicable model component rather than on an intuition component.

There is a substantial literature which suggests that forecasts based on intuition, or judgment, are typically biased. One cause of this bias is that forecasters rely on judgment when it is known that models suffer from omitted variables, see Bunn and Salo [1]. It has been found that relying on casual adjustments in practice may lead to a double-counting bias, whereas the use of model-based forecasts may be able to accommodate the omitted variables. In order to sketch one of the implications, consider an autoregressive model of order 1 [AR(1)] for the variable $X_{t}$, namely 


$$
X_{t}=\rho X_{t-1}+\varepsilon_{t}
$$

OLS will provide an estimate $\hat{\rho}$, and an unbiased forecast for $X_{n+1}$ is $\hat{\rho} X_{n}$. Suppose the forecaster adds intuition, believing that the value of $X_{n}$ is exceptional and that the forecasts need further accommodation. Adding such intuition based on $X_{n}$ makes the forecast $\hat{\rho}^{*} X_{n}$. It is straightforward to see that this expert forecast will be biased (although it may be more accurate in terms of RMSPE). Furthermore, the forecast error computed as $X_{n+1}-\hat{\rho}^{*} X_{n}$ will depend on $X_{n}$, and thereby will be predictable.

To see whether such a situation also holds for Taiwan, consider the results in Table 7. This table presents a comparison of intuition versus publicly available information in explaining the forecast errors for the inflation rate and real GDP growth rate. This table examines to what extent forecasters should rely on models or on their intuition. If publicly available information that is used judiciously in a model is able to predict the one-, two- and three-quarter forecast errors for the inflation rate and/or real GDP growth rate, intuition would be less important in explaining forecast errors than the use of an econometric model. It can be seen from Table 7 that the one-, two- and three-quarter forecast errors, A-R, A-P and A-I, respectively, for the inflation rate and real GDP growth rate, can be explained using publicly available information, specifically the lagged values of the respective variables. Thus, publicly available information is significant in explaining the forecast errors for both the inflation rate and the real GDP growth rate, which shows that the intuition part of the forecasts must also include the same regressors as in the model forecast, and hence forecasters can be seen to double count.

Thus, there is substantial room for improving the forecasts of the inflation rate and real GDP growth rate for Taiwan. Such a strategy would entail downplaying the role of intuition and relying 
more heavily on the replicable econometric model component, which would also reduce the chance of double counting.

The null hypothesis of Neutral Forecast Updates, that is, tests of equality of means between the forecast updates, is tested in Tables 8 and 9 for the inflation rate and real GDP growth rate, respectively. The null hypothesis can be expressed as H0: P-I = R-P, in which case the one-quarter forecast updates are equivalent, on average. The alternative hypothesis of Progressive Forecast Updates is H1: P-I > R-P, which states that the accuracy of forecast updates increases, on average, as the forecasts approach the actual value.

The results in Tables 8 and 9 show that the ANOVA and Welch [11] tests reject the null hypothesis of Neutral Forecast Updates for the inflation rate at the 5\% level of significance, but do not reject the null hypothesis for the real GDP growth rate. As seen in Table 3, the primary and revised forecasts are very similar for the inflation rate, whereas the initial and primary forecasts are not, so that P-I is not close to R-P. However, the three forecasts are not very close to each other for the real GDP growth rate, so that it is more likely that P-I and R-P might be equivalent. Moreover, the one-quarter forecast updates suggest that P-I > R-P, so that additional information is helpful in forecasting as the initial forecast is updated to the primary forecast, and subsequently to the revised forecast, for both the inflation rate and the real GDP growth rate.

\section{Concluding Remarks}

As macroeconomic forecasts typically involve both a model component, which is replicable, as well as intuition, which is non-replicable, it is important to differentiate between their respective contributions to obtain accurate forecasts and improved forecast updates. If forecast updates are

progressive, forecasts should become more accurate as the forecast horizon increases. Otherwise, 
forecast updates would be neutral.

The paper examined whether intuition or publicly available information was more important in explaining one-quarter forecast updates and one-, two- and three-quarter forecast errors for two economic fundamentals for Taiwan, namely the inflation rate and the real GDP growth rate, and whether intuition could reduce forecast updates and forecast errors. The one-quarter forecast update could be explained for the first update, but not the second update, for both the inflation rate and real GDP growth rate, whereas the one-, two- and three-quarter forecast errors could be explained for both the inflation rate and real GDP growth rate. As the primary and revised updates were very similar, the one-quarter forecast update based on these two forecasts could not be explained using publicly available information. It was found that forecast updates were progressive. Moreover, the one-, two- and three-quarter forecast errors could be explained using publicly available information, so that the importance of intuition was less important.

For policy purposes, the finding that forecast updates were progressive, and essentially depended on intuition, means that econometric models may not be useful in updating forecasts. However, when they did so, a greater gain in forecast accuracy was observed. As the one-, two- and three-quarter forecast errors were predictable on the basis of their own past values, it can be seen that forecasters include these same regressors again in forming their intuition, and hence are double counting. Thus, there is substantial room for improvement in the macroeconomic forecasts for Taiwan. It is strongly recommended that intuition should play a lesser role, with greater emphasis on using the available information set and an appropriate econometric model. 


\section{References}

[1] Bunn, D.W. and A.A. Salo (1996), Adjustment of forecasts with model consistent expectations, International Journal of Forecasting, 12, 163-170.

[2] Chang, C.-L., P.H. Franses and M. McAleer (2011), How accurate are government forecasts of economic fundamentals? The case of Taiwan, International Journal of Forecasting, 27(4), 1066-1075.

[3] Clark, T.E. and M.W. McCracken (2001), Tests of equal forecast accuracy and encompassing for nested models, Journal of Econometrics, 105, 85-110.

[4] Fiebig, D.G., M. McAleer and R. Bartels (1992), Properties of ordinary least squares estimators in regression models with non-spherical disturbances, Journal of Econometrics, 54, 321-334.

[5] Franses, P.H., M. McAleer and R. Legerstee (2009), Expert opinion versus expertise in forecasting, Statistica Neerlandica, 63, 334-346.

[6] McAleer, M. (1992), Efficient estimation: the Rao-Zyskind condition, Kruskal's theorem and ordinary least squares, Economic Record, 68, 65-72.

[7] McAleer, M. and C. McKenzie (1991), When are two step estimators efficient?, Econometric Reviews, 10, 235-252.

[8] Oxley, L. and M. McAleer (1993), Econometric issues in macroeconomic models with generated regressors, Journal of Economic Surveys, 7, 1-40.

[9] Pagan, A.R. (1984), Econometric issues in the analysis of regressions with generated regressors, International Economic Review, 25, 221-247.

[10] Smith, J. and M. McAleer (1994), Newey-West covariance matrix estimates for models with generated regressors, Applied Economics, 26, 635-640.

[11] Welch, B.L. (1951). On the comparison of several mean values: An alternative approach, Biometrika, 38, 330-336. 
Figure 1

Inflation rate (1980Q1-2009Q2)

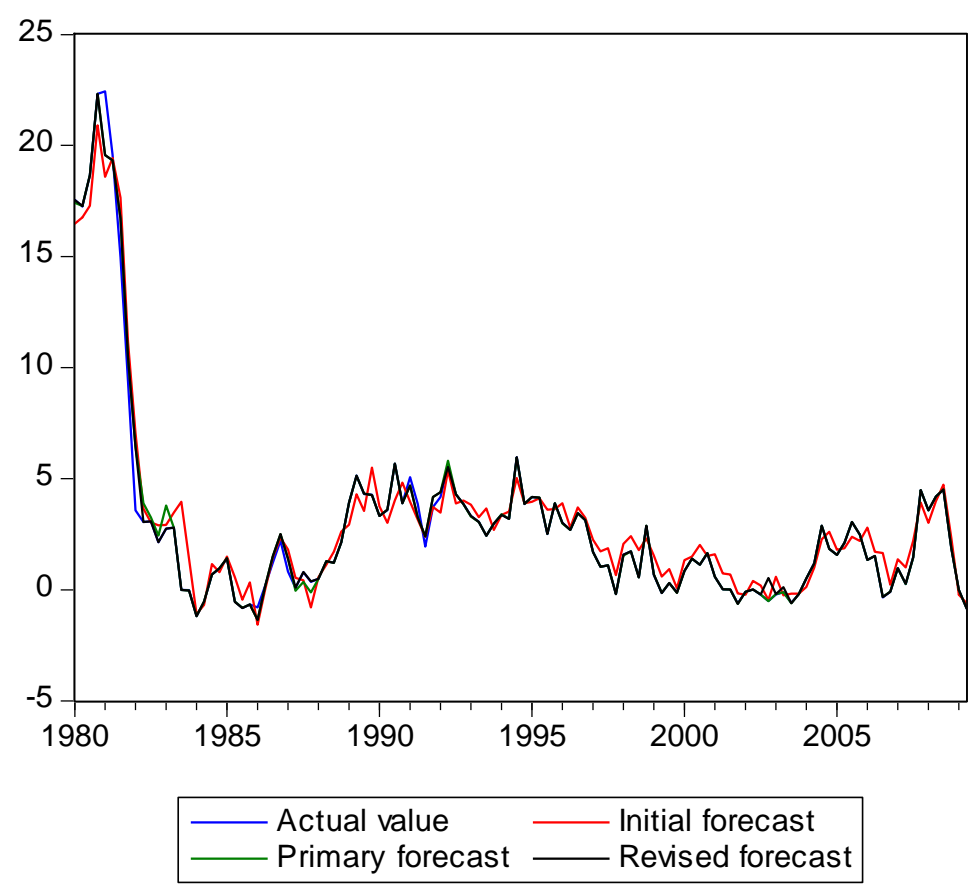


Figure 2

\section{Real GDP growth (1980Q1-2009Q2)}

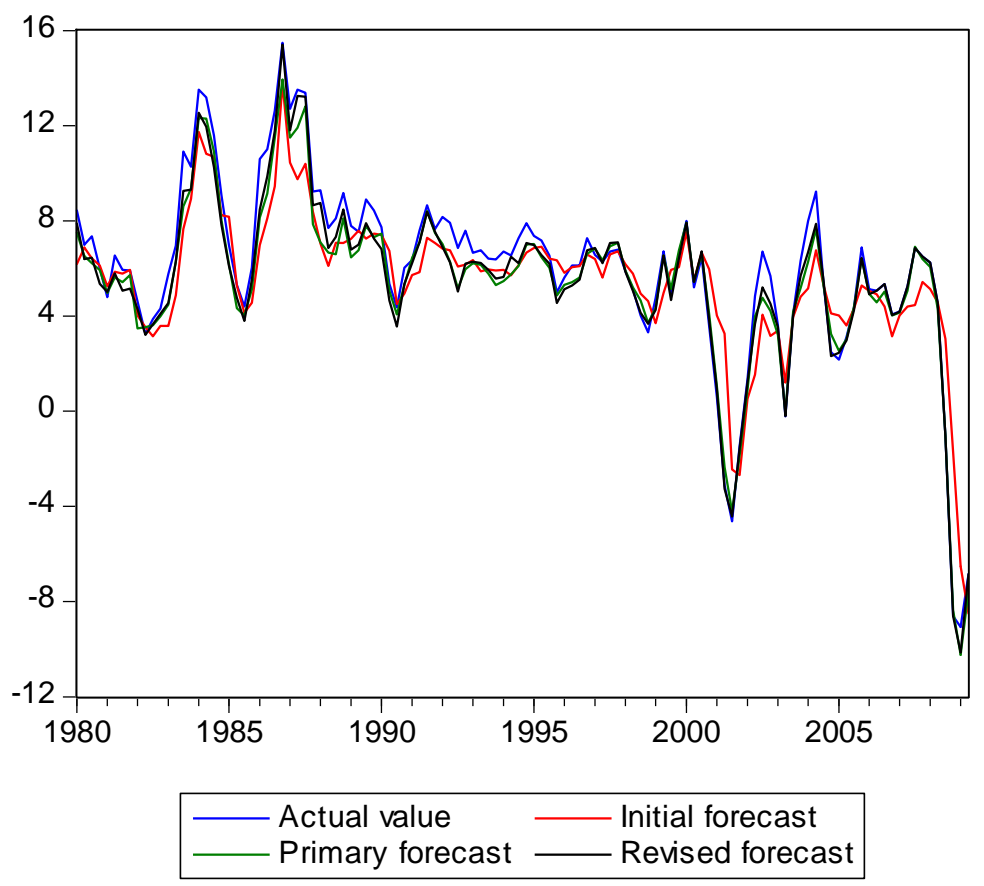


Figure 3

A-R, A-P, A-I, P-I and R-P for the Inflation Rate

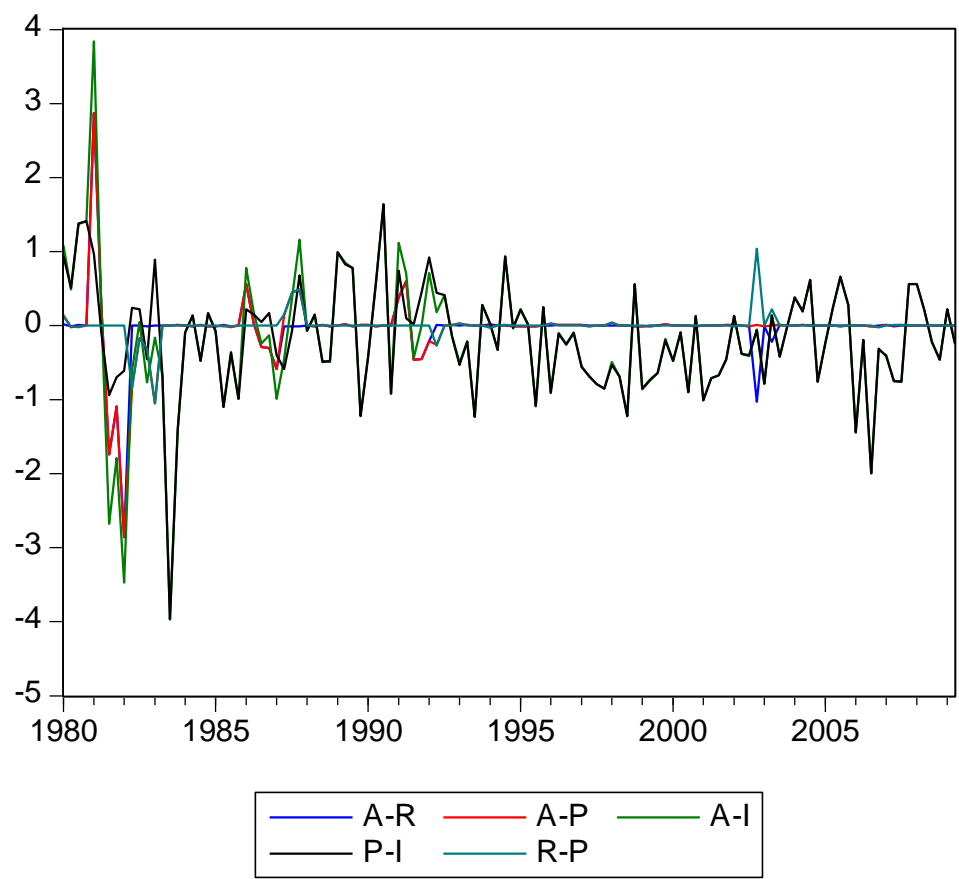

Note: I denotes initial forecast, $\mathrm{P}$ primary forecast, $\mathrm{R}$ revised forecast, and A actual value. 
Figure 4

A-R, A-P, A-I, P-I and R-P for the Real GDP Growth Rate

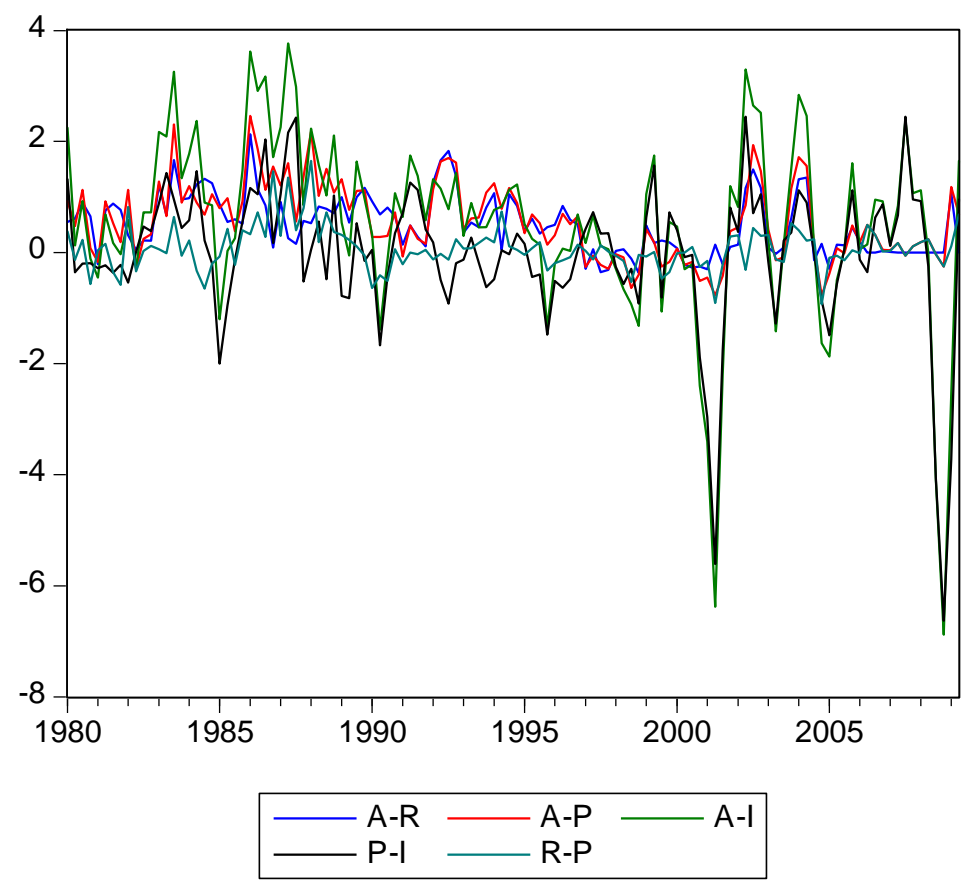

Note: I denotes initial forecast, $\mathrm{P}$ primary forecast, $\mathrm{R}$ revised forecast, and A actual value. 
Table 1

Correlations of Forecasts and Actual Value for Inflation Rate

\begin{tabular}{l|cccc}
\hline \multicolumn{1}{c|}{ Variable } & $\begin{array}{c}\text { Actual } \\
\text { value }\end{array}$ & $\begin{array}{c}\text { Initial } \\
\text { forecast }\end{array}$ & $\begin{array}{c}\text { Primary } \\
\text { forecast }\end{array}$ & $\begin{array}{c}\text { Revised } \\
\text { forecast }\end{array}$ \\
\hline Actual value & 1.000 & 0.980 & 0.995 & 0.995 \\
Initial forecast & 0.980 & 1.000 & 0.987 & 0.987 \\
Primary forecast & 0.995 & 0.987 & 1.000 & 0.999 \\
Revised forecast & 0.995 & 0.987 & 0.999 & 1.000 \\
\hline
\end{tabular}

Table 2

Correlations of Forecasts and Actual Value for Real GDP Growth Rate

\begin{tabular}{l|cccc}
\hline \multicolumn{1}{c|}{ Variable } & $\begin{array}{c}\text { Actual } \\
\text { value }\end{array}$ & $\begin{array}{c}\text { Initial } \\
\text { forecast }\end{array}$ & $\begin{array}{c}\text { Primary } \\
\text { forecast }\end{array}$ & $\begin{array}{c}\text { Revised } \\
\text { forecast }\end{array}$ \\
\hline Actual value & 1.000 & 0.917 & 0.986 & 0.991 \\
Initial forecast & 0.917 & 1.000 & 0.937 & 0.924 \\
Primary forecast & 0.986 & 0.937 & 1.000 & 0.994 \\
Revised forecast & 0.991 & 0.924 & 0.994 & 1.000 \\
\hline
\end{tabular}

Table 3

Forecast Accuracy for Inflation Rate and Real GDP Growth Rate

\begin{tabular}{l|cc|cc|}
\hline \multirow{2}{*}{ Forecast } & \multicolumn{2}{|c|}{ Inflation } & \multicolumn{2}{c|}{ Real Growth Rate } \\
\cline { 2 - 5 } Initial & RMSPE & MAD & RMSPE & MAD \\
Primary & 0.89 & 0.65 & 3.03 & 1.26 \\
Revised & 0.21 & 0.15 & 0.78 & 0.69 \\
\hline
\end{tabular}

Note: The forecast accuracy measures are root mean squared prediction error (RMSPE) and mean absolute deviation (MAD). 
Table 4

Correlations of A-R, A-P, A-I, P-I and R-P for the Inflation Rate

\begin{tabular}{c|ccccc}
\hline Variables & A-R & A-P & A-I & P-I & R-P \\
\hline A-R & 1.000 & 0.925 & 0.595 & 0.166 & -0.123 \\
A-P & 0.925 & 1.000 & 0.609 & 0.137 & 0.262 \\
A-I & 0.595 & 0.609 & 1.000 & 0.869 & 0.080 \\
P-I & 0.166 & 0.137 & 0.869 & 1.000 & -0.064 \\
R-P & -0.123 & 0.262 & 0.080 & -0.064 & 1.000 \\
\hline
\end{tabular}

Notes: I denotes initial forecast, $\mathrm{P}$ primary forecast, $\mathrm{R}$ revised forecast, and A actual value. P-I, R-P and A-R denote one-quarter forecast updates, A-P denotes a two-quarter forecast update, and A-I denotes a three-quarter forecast update.

Table 5

Correlations of A-R, A-P, A-I, P-I and R-P for the Real GDP Growth Rate

\begin{tabular}{c|ccccc}
\hline Variables & A-R & A-P & A-I & P-I & R-P \\
\hline A-R & 1.000 & 0.813 & 0.470 & 0.173 & 0.055 \\
A-P & 0.813 & 1.000 & 0.658 & 0.314 & 0.626 \\
A-I & 0.470 & 0.658 & 1.000 & 0.921 & 0.499 \\
P-I & 0.173 & 0.314 & 0.921 & 1.000 & 0.307 \\
R-P & 0.055 & 0.626 & 0.499 & 0.307 & 1.000 \\
\hline
\end{tabular}

Notes: I denotes initial forecast, $\mathrm{P}$ primary forecast, $\mathrm{R}$ revised forecast, and A actual value. P-I, R-P and A-R denote one-quarter forecast updates, A-P denotes a two-quarter forecast update, and A-I denotes a three-quarter forecast update. 
Table 6

Explaining Forecast Updates for the Inflation Rate and Real GDP Growth Rate

\begin{tabular}{|c|c|c|c|c|}
\hline & \multicolumn{2}{|c|}{ Inflation Rate } & \multicolumn{2}{|c|}{ Real Growth Rate } \\
\hline \multirow{2}{*}{ Variable } & (1) & (2) & (3) & (4) \\
\hline & P-I & $\mathrm{R}-\mathrm{P}$ & P-I & $\mathrm{R}-\mathrm{P}$ \\
\hline \multirow{3}{*}{ Constant } & -0.356 & -0.030 & -0.180 & 0.009 \\
\hline & $(0.147)^{* *}$ & $(0.036)$ & $(0.223)$ & $(0.080)$ \\
\hline & {$[0.115]^{* * *}$} & [0.043] & {$[0.252]$} & [0.125] \\
\hline \multirow{3}{*}{ AI $(-1)$} & 0.095 & 0.015 & 0.001 & -0.013 \\
\hline & $(0.046)^{* *}$ & $(0.011)$ & $(0.069)$ & $(0.024)$ \\
\hline & {$[0.037]^{* *}$} & [0.014] & {$[0.056]$} & [0.020] \\
\hline \multirow{3}{*}{ AI $(-2)$} & -0.062 & -0.019 & -0.023 & -0.0003 \\
\hline & $(0.045)$ & $(0.011)^{*}$ & $(0.068)$ & $(0.024)$ \\
\hline & [0.043] & [0.014] & {$[0.050]$} & [0.017] \\
\hline \multirow{3}{*}{ AG (-1) } & 0.008 & 0.006 & 0.397 & 0.026 \\
\hline & $(0.035)$ & $(0.009)$ & $(0.054)^{* * *}$ & $(0.019)$ \\
\hline & [0.037] & {$[0.006]$} & {$[0.091]^{* * *}$} & {$[0.026]$} \\
\hline \multirow{3}{*}{ AG (-2) } & 0.006 & 0.001 & -0.354 & -0.009 \\
\hline & $(0.038)$ & $(0.009)$ & $(0.058)^{* * *}$ & $(0.021)$ \\
\hline & [0.037] & [0.008] & {$[0.100]^{* * *}$} & [0.038] \\
\hline $\operatorname{Adj} R^{2}$ & 0.042 & 0.022 & 0.313 & 0.022 \\
\hline F-statistic & $2.283^{*}$ & 1.663 & $14.33^{* * *}$ & 1.670 \\
\hline
\end{tabular}

Notes: I denotes initial forecast, P primary forecast, R revised forecast, A actual value. P-I, $\mathrm{R}-\mathrm{P}$ and A-R denote one-quarter forecast updates, A-P a two-quarter forecast update, and A-I a three-quarter forecast update. $\mathrm{AI}(-i)$ denotes actual inflation rate lagged $i$ periods, and AG(-i) actual real GDP growth rate lagged $i$ periods, $i=1$,2. OLS standard errors are in parentheses, Newey-West HAC standard errors are in brackets. *, ** and *** denote significance at the $10 \%, 5 \%$ and $1 \%$ levels, respectively. 
Table 7

Explaining Forecast Errors for the Inflation Rate and Real GDP Growth Rate

\begin{tabular}{|c|c|c|c|c|c|c|}
\hline \multirow{3}{*}{ Variable } & \multicolumn{3}{|c|}{ Inflation Rate } & \multicolumn{3}{|c|}{ Real Growth Rate } \\
\hline & (1) & (2) & (3) & (4) & (5) & (6) \\
\hline & $A-R$ & A-P & A-I & $A-R$ & A-P & A-I \\
\hline \multirow[t]{3}{*}{ Constant } & 0.068 & 0.038 & -0.318 & 0.280 & 0.289 & 0.109 \\
\hline & $(0.082)$ & $(0.082)$ & $(0.175)^{*}$ & $(0.104)^{* * *}$ & $(0.132)^{* *}$ & $(0.280)$ \\
\hline & {$[0.051]$} & {$[0.056]$} & {$[0.148]^{* *}$} & {$[0.130]^{* *}$} & {$[0.205]$} & [0.383] \\
\hline \multirow[t]{3}{*}{ AI (-1) } & 0.137 & 0.152 & 0.247 & 0.021 & 0.008 & 0.009 \\
\hline & $(0.025)^{* * *}$ & $(0.025)^{* * *}$ & $(0.054)^{* * *}$ & $(0.032)$ & $(0.041)$ & $(0.086)$ \\
\hline & {$[0.070]^{*}$} & {$[0.074]^{* *}$} & {$[0.094]^{* *}$} & [0.032] & [0.035] & {$[0.074]$} \\
\hline \multirow[t]{3}{*}{ AI (-2) } & -0.140 & -0.159 & -0.220 & -0.013 & -0.013 & -0.036 \\
\hline & $(0.025)^{* * *}$ & $(0.025)^{* * *}$ & $(0.053)^{* * *}$ & $(0.032)$ & $(0.040)$ & $(0.085)$ \\
\hline & {$[0.069]^{* *}$} & {$[0.073]^{* *}$} & {$[0.102]^{* *}$} & [0.029] & [0.033] & [0.068] \\
\hline \multirow[t]{3}{*}{$A G(-1)$} & -0.026 & -0.021 & -0.012 & 0.076 & 0.102 & 0.499 \\
\hline & $(0.020)$ & $(0.020)$ & $(0.042)$ & $(0.025)^{* * *}$ & $(0.032)^{* * *}$ & $(0.067)^{* * *}$ \\
\hline & {$[0.018]$} & {$[0.017]$} & {$[0.042]$} & {$[0.034]^{* *}$} & {$[0.052]^{* *}$} & {$[0.086]^{* * *}$} \\
\hline \multirow[t]{3}{*}{$A G(-2)$} & 0.013 & 0.014 & 0.019 & -0.044 & -0.053 & -0.408 \\
\hline & $(0.021)$ & $(0.021)$ & $(0.046)$ & $(0.027)$ & $(0.034)$ & $(0.073)^{* * *}$ \\
\hline & {$[0.016]$} & {$[0.016]$} & {$[0.043]$} & [0.034] & [0.054] & {$[0.115]^{* * *}$} \\
\hline $\operatorname{Adj} R^{2}$ & 0.200 & 0.245 & 0.139 & 0.079 & 0.098 & 0.323 \\
\hline F-statistic & $8.331 * * *$ & $10.51 * * *$ & $5.707 * * *$ & $3.509 * * *$ & $4.170^{* * *}$ & $14.96 * * *$ \\
\hline
\end{tabular}

Notes: I denotes initial forecast, $\mathrm{P}$ primary forecast, R revised forecast, A actual value. P-I, R-P and A-R denote one-quarter forecast updates, A-P a two-quarter forecast update, and A-I a three-quarter forecast update. AI(-i) denotes actual inflation rate lagged $i$ periods, and AG(- $-i)$ actual real GDP growth rate lagged $i$ periods, $i=1$,2. OLS standard errors are in parentheses, Newey-West HAC standard errors are in brackets. *, ** and *** denote significance at the $10 \%, 5 \%$ and $1 \%$ levels, respectively. 
Table 8

Test of Neutral Forecast Updates for Inflation Rate 1980Q1-2009Q2

\begin{tabular}{l|ccc}
\hline Method & df. & Value & Probability \\
\hline t-test & 234 & -2.485 & 0.014 \\
Welch F-test* & $1,130.28$ & 6.173 & 0.014 \\
\hline
\end{tabular}

Test for Equality of Means Ho: P-I=R-P

Included observations: 118

*The Welch [11] test allows unequal cell variances.

Table 9

Test of Neutral Forecast Updates for Real GDP Growth Rate 1980Q1-2009Q2

\begin{tabular}{l|ccc}
\hline Method & df. & Value & Probability \\
\hline t-test & 234 & -0.896 & 0.371 \\
Welch F-test* & $1,138.06$ & 0.802 & 0.372 \\
\hline
\end{tabular}

Test for Equality of Means Ho: P-I=R-P

Included observations: 118

*The Welch [11] test allows unequal cell variances. 